International Journal of Pure and Applied Mathematics

Volume 115 No. $4 \quad 2017,873-881$

ISSN: 1311-8080 (printed version); ISSN: 1314-3395 (on-line version)

url: http://www.ijpam.eu

doi: 10.12732/ijpam.v115i4.21

ijpam.eu

\title{
A NOTE ABOUT STABILITY OF FRACTIONAL RETARDED LINEAR SYSTEMS WITH DISTRIBUTED DELAYS
}

\author{
Marian Milev ${ }^{1}$, Stoyan Zlatev ${ }^{2} \S$ \\ ${ }^{1}$ Department of Informatics and Statistics \\ University of Food Technology \\ 26 Maritsa Blvd., 4000 Plovdiv, BULGARIA \\ ${ }^{2}$ Faculty of Mathematics and Informatics \\ University of Plovdiv \\ 4 Tzar Asen, 4000 Plovdiv, BULGARIA
}

\begin{abstract}
The main goal of this paper is to establish computational sufficient conditions for global asymptotic stability for a class of retarded linear fractional differential systems with distributed delays.

In the work are considered both cases - when the derivatives in the system are in RiemannLiouville or Caputo type.
\end{abstract}

AMS Subject Classification: $34 \mathrm{~A} 08,34 \mathrm{~A} 12,34 \mathrm{D} 20$

Key Words: fractional derivatives, distributed delay, fractional differential system, logarithmic norm

\section{Introduction}

It is well known that the fractional calculus is a subject that has gained considerably popularity and importance in the past few decades in diverse fields of science and engineering. A good overview in this direction can be received from the monographs [5], [6], [10] and [4]. As far as we know the first overview of

Received: $\quad$ May 20, 2017

Revised: $\quad$ August 1, 2017

Published: August 9, 2017

${ }^{\S}$ Correspondence author (c) 2017 Academic Publications, Ltd. url: www.acadpubl.eu 
the results concerning the stability analysis of fractional differential equations is the survey [7]. For linear fractional system with single order derivatives and constant delays we point out the works [3] and [15]. The case of distributed delays for retarded type fractional system of is studied in [11], [14] and for neutral systems in [13] and [12]. Some stability results for fractional system with distributed order derivatives and constant delays are given in [9] and the case of distributed delays is considered in [1]. The works [11] and [13] are our first motivation to extend and improve some of the obtained there explicit type sufficient conditions for asymptotical stability of the zero solution of this systems.

The main goal of this work is to establish sufficient explicit conditions for globally asymptotic stability of linear fractional differential system with distributed delays. Under some natural additional conditions we replace part of the complicated for practical checking conditions with significantly simple task - to study the distribution of the eigenvalues of a so called "associated characteristic matrix".

The paper is organized as follows: In Section 2, we recall some needed definitions about Riemann-Liouville and Caputo fractional derivatives as well as some results about the matrix measure (named also Lozinskii measure [8] or logarithmic norm [2])for square complex matrices. Section 3 is devoted to the statement of the Cauchy problem for linear incommensurate fractional differential system with distributed delays in the cases of Riemann-Liouville and Caputo derivatives. In addition we prove a technical lemma which we will use in the next section. In Section 4 using the matrix measure technique we establish some explicit type sufficient conditions for global asymptotical stability of the considered fractional differential system with distributed delays.

\section{Preliminaries}

As preliminaries we recall the definitions of Riemann-Liouville and Caputo fractional derivatives (left and right side) as well as some results about the logarithmic norm (named also matrix measure or Lozinskii measure) for square complex matrices.

We will use following notations: $\mathbb{R}_{+}=(0, \infty) ; \overline{\mathbb{R}}_{+}=[0, \infty) ; \mathbb{C}_{+}=\{p \in$ $\mathbb{C} \mid$ Re $p>0\} ; \overline{\mathbb{C}}_{+}=\{p \in \mathbb{C} \mid \operatorname{Rep} \geq 0\} ; \mathbb{C}_{-}=\mathbb{C} \backslash \overline{\mathbb{C}}_{+} ; \mathbb{N}_{0}=\mathbb{N} \cup\{0\} ; \mathbb{N}_{\infty}=$ $\mathbb{N} \cup\{\infty\}$ and $\langle n\rangle=\{1,2, \ldots, n\}$. With $L_{1}^{\text {loc }}(\mathbb{R}, \mathbb{R})$ we denote the linear space of all locally Lebesgue integrable functions $f: \mathbb{R} \rightarrow \mathbb{R}$.

For each $a \in \mathbb{R}$ and $f \in L_{1}^{\text {loc }}(\mathbb{R}, \mathbb{R})$ the left and right side Riemann-Liouville 
fractional derivative for $\alpha \in(0,1)$ are defined by

$$
\begin{gathered}
R L D_{a+}^{\alpha} f(t)=\frac{1}{\Gamma(1-\alpha)} \frac{d}{d t} \int_{a}^{t}(t-s)^{-\alpha} f(s) d s, t>a \\
R L D_{a-}^{\alpha} f(t)=-\frac{1}{\Gamma(1-\alpha)} \frac{d}{d t} \int_{t}^{a}(s-t)^{-\alpha} f(s) d s, t<a .
\end{gathered}
$$

The Caputo fractional left and right side derivative are defined for $\alpha \in(0,1)$ by the equalities

$$
\begin{aligned}
& C D_{a+}^{\alpha} f(t)={ }_{R L} D_{a+}^{\alpha}[f(s)-f(a)](t), t>a \\
& C D_{a-}^{\alpha} f(t)={ }_{R L} D_{a-}^{\alpha}[f(s)-f(a)](t), t<a .
\end{aligned}
$$

Let $C=\left\{c_{k}^{j}\right\}_{k, j=1}^{n} \in \mathbb{C}^{n \times n}$ and denote with $S p(C)$ the spectrum of $C$. As usual $\|z\|_{p}=\left(\sum_{p=1}^{n}\left|z_{i}\right|^{p}\right)^{\frac{1}{p}},\|z\|_{\infty}=\max _{1 \leq k \leq n}\left\{\left|z_{k}\right|\right\}$ and $\|C\|_{p}=\max _{\|z\|_{p}=1}\|C z\|_{p}, z \in$ $\mathbb{C}^{n}, p \in \mathbb{N}_{\infty}$.

Definition 1. ([8], [2]) The function $\mu_{p}: \mathbb{C}^{n \times n} \rightarrow \mathbb{R}, p \in \mathbb{N}_{\infty}$ defined with the equality $\mu_{p}(C)=\lim _{\varepsilon \rightarrow+0} \varepsilon^{-1}\left(\|I+\varepsilon C\|_{p}-1\right)$, where $C \in \mathbb{C}^{n \times n}, I \in \mathbb{C}^{n \times n}$ is the unit matrix, $\varepsilon \in \mathbb{R}_{+}$is called matrix measure (named also Lozinskii measure or logarithmic norm).

For a good overview over the properties of the matrix measure we refer to [8], [2]. For our considerations below we recall that $\mu_{\infty}(C)=\sup _{1 \leq k \leq n}\{R e$ $\left.c_{k}^{k}+\sum_{j=1, j \neq k}^{n}\left|c_{k}^{j}\right|\right\}$ and $\mu_{p}(C) \geq \operatorname{Re} \lambda \geq-\mu_{p}(-C)$ for each $\lambda \in \operatorname{Sp}(A)$.

Obviously from the last inequalities it follows that all eigenvalues of any matrix $C=\left\{c_{k}^{j}\right\}_{k, j=1}^{n} \in \mathbb{C}^{n \times n}$ with $\mu_{p}(C)<0$ for some $p \in \mathbb{N}_{\infty}$ have negative real parts.

\section{Problem Statement}

Consider the following autonomous linear systems with distributed delay

$$
D_{0+}^{\alpha_{k}} x_{k}(t)=\sum_{j=1}^{n} \int_{-\sigma}^{0} x_{j}(t+\theta) d u_{k}^{j}(\theta), k \in\langle n\rangle
$$


where $D_{0+}^{\alpha_{k}}$ denotes either ${ }_{R L} D_{0+}^{\alpha_{k}}$ (Riemann-Liouville fractional derivative) or ${ }_{C} D_{0 \pm}^{\alpha_{k}}$ (Caputo fractional derivative), $\alpha_{k} \in(0,1)$,

$\sigma \in \mathbb{R}_{+}=[0, \infty)$.

We denote with: $X(t)=\left(x_{1}(t), \ldots, x_{n}(t)\right)^{T},|X(t)|=\sum_{k=1}^{n}\left|x_{k}(t)\right|$,

$\alpha_{m}=\min \left(\alpha_{1}, \ldots, \alpha_{n}\right), \alpha_{M}=\max \left(\alpha_{1}, \ldots, \alpha_{n}\right)$,

${ }_{R L} D_{0+}^{\alpha} X(t)=\left({ }_{R L} D_{0+}^{\alpha_{1}} x_{1}(t), \ldots, R L D_{0+}^{\alpha_{n}} x_{n}(t)\right)^{T}$,

${ }_{C} D_{0+}^{\alpha} X(t)=\left({ }_{C} D_{0+}^{\alpha_{1}} x_{1}(t), \ldots,{ }_{C} D_{0+}^{\alpha_{n}} x_{n}(t)\right)^{T}$ for each $t \in \mathbb{R}_{+}$. For $W: \mathbb{R} \times \mathbb{R} \rightarrow$ $\mathbb{R}^{n \times n}, W(\theta)=\left\{w_{j}^{i}(\theta)\right\}_{i, j=1}^{n}$ we denote

$|W(\theta)|=\sum_{k, j=1}^{n}\left|w_{k}^{j}(\theta)\right|$ and by $B V[a, b]$ the linear space of functions with bounded variation in $\theta$ on $[a, b] \subset \mathbb{R}, a \leq b$, where

$\operatorname{Var}_{[a, b]} W(\cdot)=\sum_{k, j=1}^{n} \operatorname{Var}_{[a, b]} w_{k}^{j}(\cdot)$.

We say that for the kernel $U: \mathbb{R} \rightarrow \mathbb{R}^{n \times n}$ the conditions (S) are fulfilled if the following conditions hold:

(S1) The function $U \in B V[-\sigma, 0]$ and is normalized so that $U(0)=0, U(\theta)=$ 0 for $\theta \geq 0, U(\theta)=U(-\sigma)$ for $\theta \leq-\sigma$.

(S2) The Lebesgue decomposition for the kernel $U(\theta)$ for $\theta \in[-\sigma, 0]$ has the form:

$U(\theta)=\aleph(\theta)+\int_{-\sigma}^{\theta} B(s) d s+\Im(\theta)$,

where $\aleph(\theta)=\left\{a_{k}^{j} H\left(\theta+\sigma_{k}^{j}\right)\right\}_{k, j=1}^{n}, \sigma_{k}^{j} \in[0, \sigma], A=\left\{a_{k}^{j}\right\}_{k, j=1}^{n} \in \mathbb{R}^{n \times n}, H(t)$ is the Heaviside function,

$\int_{-\sigma}^{\theta} B(s) d s=\left\{\int_{-\sigma}^{\theta} b_{k}^{j}(s) d s\right\}_{k, j=1}^{n} \in A C\left([-\sigma, 0], \mathbb{R}^{n \times n}\right)$ and the singular part in the decomposition $\Im(\theta)=\left\{g_{k}^{j}(\theta)\right\}_{k, j=1}^{n} \in C\left([-\sigma, 0], \mathbb{R}^{n \times n}\right)$, $k, j \in\langle n\rangle$.

We consider first the case when the derivatives in the system (3.1) are in Riemann-Liouville sense and define an initial value problem (IVP) for (3.1) under the following initial condition:

$$
D_{0-}^{\alpha_{k}-1} x_{k}(t)=\phi_{k}(t), t \in[-\sigma, 0], \Phi \in \mathfrak{C}, k \in\langle n\rangle,
$$

where $\mathfrak{C}=\left\{\Phi:[\sigma, 0] \rightarrow \mathbb{R}^{n} \mid \Phi(t)=\left(\phi_{1}(t), \ldots, \phi_{n}(t)\right)^{T}, \phi_{k} \in C([-\sigma, 0], \mathbb{R}), k \in\right.$ $\langle n\rangle\}$ with the norm $\|\Phi\|=\sup _{t \in[-\sigma, 0]} \sum_{k=1}^{n}\left|\phi_{k}(t)\right|=\sup _{t \in[-\sigma, 0]}|\Phi(t)|$ is a Banach space of initial vector functions.

For the case when in the system (3.1) the derivatives are Caputo sense then 
the IVP problem for (3.1) will be defined under the standard initial condition:

$$
X(t)=\Phi(t), t \in[-\sigma, 0], \Phi \in \mathfrak{C}
$$

For all questions about the existing, uniqueness and continuation on $\mathbb{R}_{+}$of the solutions of the IVP problems (3.1), (3.2) and (3.1), (3.3) we refer [11], [13] and [12]. Note that in [12] is studied also when for the system (3.1) can be applied correct the Laplace transform.

For our calculation below we specify a single-valued branch for $p \in \mathbb{C} \backslash$ $(-\infty, 0],-\pi<\arg p<\pi$ from the multiple-valued function $p^{\alpha}$. Moreover this branch can be prolonged at $p=0$ as continuous in $\overline{\mathbb{C}}_{+}$function. Note that at $p=0$ this branch will be only continuous but not entire function.

Definition 2. [11] For the homogenous system (3.1) we call the matrix valued function

$$
G(p)=\left(\begin{array}{ccc}
p^{\alpha_{1}}-\int_{-\sigma}^{0} e^{p \theta} d u_{1}^{1}(\theta) & \ldots & -\int_{-\sigma}^{0} e^{p \theta} d u_{1}^{n}(\theta) \\
\ldots & & p^{\alpha_{n}}-\int_{-\sigma}^{0} e^{p \theta} d u_{n}^{n}(\theta)
\end{array}\right)
$$

characteristic matrix and the equation

$$
\operatorname{det} G(p)=0
$$

characteristic equation.

Definition 3. [13] For the homogenous system (3.1) the matrix valued function

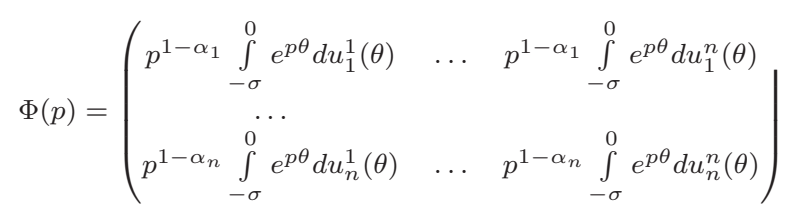

will be called associated characteristic matrix (ACM).

The next lemma locates the roots with nonnegative real parts of the characteristic equation (3.5) in $\overline{\mathbb{C}}_{+}$.

Lemma 4. [[13], Lemmas 5 and 6] Let the following conditions be fulfilled:

1. The conditions S hold.

2. $\operatorname{Var}_{[-\sigma, 0]} U(\cdot)>0$. 
Then all roots of the characteristic equation (3.5) with nonnegative real parts belong to the rectangle

$$
\Omega=\left\{p=\gamma+i \omega \in \overline{\mathbb{C}}_{+}\left|0 \leq \gamma \leq s_{*}, 0 \leq\right| \omega \mid \leq s_{*}\right\},
$$

where $s_{*} \in \mathbb{R}$ is the unique root in $\mathbb{R}_{+}$of the equation

$$
h(s)=\min \left(s^{\alpha_{m}}, s^{\alpha_{M}}\right)-\operatorname{Var}_{[-\sigma, 0]} U(\cdot)=0
$$

Lemma 5. [[13], Lemma 4] A complex number $p_{*} \in \overline{\mathbb{C}}_{+}, p_{*} \neq 0$ is an eigenvalue for the matrix $\Phi\left(p_{*}\right)$ if and only if when $p_{*}$ is a root of the characteristic equation (3.5).

Lemma 6. Let the following conditions are fulfilled:

1. The conditions of Lemma 4 hold.

2. The functions $u_{k}^{k}(\theta)$ are monotone decreasing on $[-\sigma, 0]$ for each $k \in\langle n\rangle$ and $\prod_{k=1}^{n} u_{k}^{k}(-\sigma)>0$.

3. $2 \sigma s_{*}<\pi$ where $s_{*} \in \mathbb{R}_{+}$is the unique root in $\mathbb{R}_{+}$of the equation (3.7).

Then for each $k \in\langle n\rangle$ and $p \in \Omega \backslash\{0\}$ the inequality

$$
\operatorname{Re}\left[p^{1-\alpha_{k}} \int_{-\sigma}^{0} e^{p \theta} d u_{k}^{k}(\theta)\right]<0
$$

holds.

Proof. For each $k \in\langle n\rangle$ and $p \in \mathbb{C} \backslash(-\infty, 0]$ we have that

$$
\begin{gathered}
p^{1-\alpha_{k}} \int_{-\sigma}^{0} e^{p \theta} d u_{k}^{k}(\theta)=|p|^{1-\alpha_{k}}\left(\cos \left(1-\alpha_{k}\right) \varphi+\right. \\
\left.+i \sin \left(1-\alpha_{k}\right) \varphi\right) \int_{-\sigma}^{0} e^{\gamma \theta}(\cos \theta \omega+i \sin \theta \omega) d u_{k}^{k}(\theta)
\end{gathered}
$$


where $\varphi=\arg \quad p \in(-\pi, \pi)$. From (3.8) for each $k \in\langle n\rangle$ and $p \in \Omega \backslash\{0\}$ it follows that

$$
\begin{gathered}
\operatorname{Re}\left[p^{1-\alpha_{k}} \int_{-\sigma}^{0} e^{p \theta} d u_{k}^{k}(\theta)\right]= \\
=|p|^{1-\alpha_{k}} \int_{-\sigma}^{0} e^{\gamma \theta} \cos \left[\left(1-\alpha_{k}\right) \varphi+\theta \omega\right] d u_{k}^{k}(\theta) .
\end{gathered}
$$

For $p \in \Omega \backslash\{0\}$ with $\omega \geq 0$ and $\theta \in[-\sigma, 0]$ we have that

$$
\left(1-\alpha_{k}\right) \varphi+\theta \omega \leq\left(1-\alpha_{m}\right) \varphi \leq\left(1-\alpha_{m}\right) \frac{\pi}{2}<\frac{\pi}{2}
$$

Taking into account condition 3 of Lemma 6 we obtain that

$$
\left(1-\alpha_{k}\right) \varphi+\theta \omega \geq-\sigma \omega \geq-\sigma s_{*}>-\frac{\pi}{2}
$$

Then from (3.10) and (3.11) for $p \in \Omega \backslash\{0\}$ with $\omega \geq 0$ and $\theta \in[-\sigma, 0]$ it follows that

$$
\frac{\pi}{2}>\left(1-\alpha_{k}\right) \varphi+\theta \omega>-\frac{\pi}{2}
$$

for each $k \in\langle n\rangle$. Since $\cos \left(\left(1-\alpha_{k}\right) \varphi+\theta \omega\right)$ is a real valued and even function then we can conclude that for $p \in \Omega \backslash\{0\}$, with $\omega \leq 0$ and $\theta \in[-\sigma, 0]$ the inequalities (3.12) hold for each $k \in\langle n\rangle$ too.

Let $p \in \Omega \backslash\{0\}$ be an arbitrary point. Then (3.12) implies that $\cos [(1-$ $\left.\left.\alpha_{k}\right) \varphi+\theta \omega\right] \geq \cos \sigma s_{*}>0$ for each $k \in\langle n\rangle$ and hence from (3.9) we obtain

$$
\begin{aligned}
& \operatorname{Re}\left[p^{1-\alpha_{k}} \int_{-\sigma}^{0} e^{p \theta} d u_{k}^{k}(\theta)\right]= \\
& \quad=-|p|^{1-\alpha_{k}} \int_{-\sigma}^{0} e^{\gamma \theta} \cos \left[\left(1-\alpha_{k}\right) \varphi+\theta \omega\right] d\left(-u_{k}^{k}(\theta)\right) \leq \\
& \quad \leq-|p|^{1-\alpha_{k}} e^{-s_{*} \sigma} u_{k}^{k}(-\sigma) \cos \sigma s_{*}<0 .
\end{aligned}
$$




\section{Main Result}

Definition 7. The system (3.1) is said to be globally asymptotically stable (GAS) iff for every initial function $\Phi \in \mathfrak{C}$ for the corresponding solution $X(t)$ we have that $\lim _{t \rightarrow \infty}|X(t)|=0$.

Theorem 8. Let the following conditions are fulfilled:

1. The conditions of Lemma 6 hold and $G(0)>0$.

2. $e^{-s_{*} \sigma} u_{k}^{k}(-\sigma) \cos \sigma s_{*}>\sum_{j=1, j \neq k}^{n} \operatorname{Var}_{[-\sigma, 0]} u_{k}^{j}(\cdot)$ for each $k \in\langle n\rangle$.

Then the system (3.1) with derivatives in Riemann-Liouville (or Caputo) sense is GAS.

Proof. Let $p_{*} \in \Omega \backslash\{0\}$ be an arbitrary point. Then since $\left|p_{*}\right|^{1-\alpha_{k}}>0$, condition 2 of Theorem 8 implies that the following inequality holds

$$
\begin{aligned}
& -\left|p_{*}\right|^{1-\alpha_{k}} e^{-s_{*} \sigma} u_{k}^{k}(-\sigma) \cos \sigma s_{*}< \\
& <-\left|p_{*}\right|^{1-\alpha_{k}} \sum_{j=1, j \neq k}^{n} \operatorname{Var}_{[-\sigma, 0]} u_{k}^{j}(\cdot) .
\end{aligned}
$$

From (4.1) for every $p_{*} \in \Omega \backslash\{0\}$ it follows that

$$
\begin{aligned}
& \mu_{\infty}\left(\Phi\left(p_{*}\right)\right)=\max _{k \in\langle n\rangle}\left(\operatorname{Re}\left[p_{*}^{1-\alpha_{k}} \int_{-\sigma}^{0} e^{p \theta} d u_{k}^{k}(\theta)\right]+\right. \\
& \left.+\left|p_{*}\right|^{1-\alpha_{k}} \sum_{j=1, j \neq k}^{n}\left|\int_{-\sigma}^{0} e^{p_{*} \theta} d u_{k}^{j}(\theta)\right|\right) \leq \\
& \leq-\left|p_{*}\right|^{1-\alpha_{k}} \\
& \left(e^{-s_{*} \sigma} u_{k}^{k}(-\sigma) \cos \sigma s_{*}-\sum_{j=1, j \neq k}^{n} \operatorname{Var}_{[-\sigma, 0]} u_{k}^{j}(\cdot)\right)<0 .
\end{aligned}
$$

Then we can conclude that all eigenvalues of the matrix $\Phi\left(p_{*}\right)$ which are different from zero have negative real parts. Therefore since $\operatorname{det} G(0)>0$ taking into account Lemma 5 and Lemma 6 we can conclude that all roots of the characteristic equation (3.5) belong to $\mathbb{C}_{-}$. Then the statement of Theorem 8 follows from Theorem 4 in [13] for the Riemann-Liouville case or from Theorem 5 in [13] for the Caputo case. 


\section{References}

[1] D. Boyadzhiev, M. Veselinova, H. Kiskinov, A. Zahariev, Stability analysis of linear distributed order fractional systems with distributed delays, Fract. Calc. Appl. Anal., 20, No 4 (2017), 914-935, doi: 10.1515/fca-2017-0048.

[2] G. Dahlquist, Stability and Error Bounds in the Numerical Integration of Ordinary Differential Equations, Diss. 1958; reprinted in Trans. Royal Inst. of Technology, No. 130, Stockholm, 1959.

[3] W. Deng, Ch. Li, J. Lu, Stability analysis of linear fractional differential system with multiple time delays, Nonlinear Dyn., 48, (2007), 409-416.

[4] R. Herrmann, Fractional Calculus An Introduction for Physicists, 2nd Edition, World Scientific Publishing Co. Pte. Ltd., 2014.

[5] A. Kilbas, H. Srivastava, J. Trujillo, Theory and Applications of Fractional Differential Equations, Elsevier Science B.V, Amsterdam, 2006.

[6] V. Kiryakova, Generalized Fractional Calculus and Applications, Longman Scientific and Technical, Harlow, copublished in the United States with John Wiley and Sons, Inc., New York, 1994.

[7] C. Li, F. Zhang, A survey on the stability of fractional differential equations, Eur. Phys. J. Special Topics, 193 (2011), 27-47.

[8] S.M. Lozinskii, Error estimate for numerical integration of ordinary differential equations, Izv. Vyssh. Uchebn. Zaved. Mat., No. 5 (1958), 52-90.

[9] H. Saberi Najafi, A. Refahi Sheikhani, and A. Ansari, Stability analysis of distributed order fractional differential equations, Abstract and Applied Analysis, Article ID 175323, 12 pages (2011), doi: 10.1155/2011/175323.

[10] I. Podlubny, Fractional Differential Equation, Academic Press, San Diego, 1999.

[11] M. Veselinova, H. Kiskinov, A. Zahariev, Stability analysis of linear fractional differential system with distributed delays, AIP Conference Proceedings. 1690, 040013 (2015), doi: $10.1063 / 1.4936720$.

[12] M. Veselinova, H. Kiskinov, A. Zahariev, Stability analysis of neutral linear fractional system with distributed Delays, Filomat 30, No. 3 (2016), 841-851, doi: 10.2298/FIL1603841V.

[13] M. Veselinova, H. Kiskinov, A. Zahariev, Explicit conditions for stability of neutral linear fractional system with distributed delays, AIP Conference Proceedings 1978, 040005 (2016), doi: 10.1063/1.4968458.

[14] M. Veselinova, H. Kiskinov, A. Zahariev, About stability conditions for retarded fractional differential systems with distributed delays, Communications in Applied Analysis, 20 (2016), 325-334.

[15] L. Xiong, Y. Zhao, T. Jiang, Stability analysis of linear fractional order neutral system with multiple delays by algebraic approach, World Academy of Science, Engineering and Technology, 5, No. 4 (2011), 758-761. 
